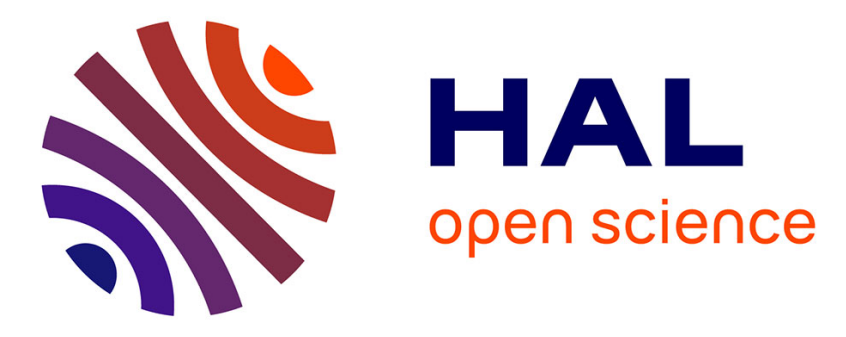

\title{
CCN-TV: a data-centric approach to real-time video services
}

Vincenzo Ciancaglini, Giuseppe Piro, Riccardo Loti, Luigi Liquori, Luigi Alfredo Grieco

\section{- To cite this version:}

Vincenzo Ciancaglini, Giuseppe Piro, Riccardo Loti, Luigi Liquori, Luigi Alfredo Grieco. CCN-TV: a data-centric approach to real-time video services. 27th International Conference on Advanced Information Networking and Applications Workshops, AINA 2013, Barcelona, Spain, March 25-28, 2013, Mar 2013, Barcelona, Spain. pp.982-989, 10.1109/WAINA.2013.19 . hal-00906830

\section{HAL Id: hal-00906830 https://hal.inria.fr/hal-00906830}

Submitted on 20 Nov 2013

HAL is a multi-disciplinary open access archive for the deposit and dissemination of scientific research documents, whether they are published or not. The documents may come from teaching and research institutions in France or abroad, or from public or private research centers.
L'archive ouverte pluridisciplinaire HAL, est destinée au dépôt et à la diffusion de documents scientifiques de niveau recherche, publiés ou non, émanant des établissements d'enseignement et de recherche français ou étrangers, des laboratoires publics ou privés. 


\title{
CCN-TV: a data-centric approach to real-time video services
}

\author{
Vincenzo Ciancaglini*, Giuseppe Piro ${ }^{\dagger}$, Riccardo Loti*, Luigi Alfredo Grieco ${ }^{\dagger}$ and Luigi Liquori* \\ *INRIA - Sophia Antipolis (France) \\ Email: \{vincenzo.ciancaglini, riccardo.loti, luigi.liquori\}@inria.fr \\ ${ }^{\dagger}$ DEE - Politecnico di Bari (Italy) \\ Email: \{g.piro,a.grieco\}@poliba.it
}

\begin{abstract}
Content-Centric Networking (CCN) is a promising data-centric architecture, based on in-network caching, name-driven routing, and receiver-initiated sessions, which can greatly enhance the way Internet resources are currently used, making support for a broader set of users with increasing traffic demands possible. The $\mathrm{CCN}$ vision is, currently, attracting the attention of many researchers across the world, since it has all the potential to become ready to the market, to be gradually deployed in the Internet of today, and to facilitate a graceful transition from a host-centric networking rationale to a more effective data-centric working behaviour. At the same time, several issues have to be investigated before $\mathrm{CCN}$ can be safely deployed at the Internet scale. They include routing, congestion control, caching operations, name-space planning, and application design. With reference to application-related facets, it is worth noticing that the demand for TV services is growing at an exponential rate over time, thus requiring a very careful analysis of their performance in $\mathrm{CCN}$ architectures. To this end, in the present contribution we deploy a CCNTV system, capable of delivering real-time streaming TV services, and we evaluate its performance through a simulation campaign based on real-world topologies.
\end{abstract}

Keywords-Content-Centric Networking, Multimedia Services, Live Streaming

\section{INTRODUCTION}

Due to the relevant importance that content sharing applications are going to play in the upcoming future [2], [3], the Content Centric Networking (CCN) rationale [4] has been proposed as a possible way to drive the current host-centric Internet paradigm towards a novel content-centric behaviour. It is based on in-network caching operations, receiver initiated data exchange, hierarchical content naming, and native support to security and privacy.

In a $\mathrm{CCN}$, contents are split in chunks which are requested using opposite Interest messages, generated at the client side. Each Interest is then routed until it reaches a node which has, in its own cache, a copy of the requested item. Then, this copy is sent, as a Data message, back along the path the Interest had gone through. Intermediate nodes can cache the Data before forwarding it to the next node (more details on the $\mathrm{CCN}$ working behavior is provided in Sec. II).

Since its born, the $\mathrm{CCN}$ vision has gained a warm attention from both scientific and industrial communities to discover the bounds of its real potential from different perspectives. Many studies have focused on modeling and designing caching strategies and data-transfer performance such as in [5]-[10]. In that direction, it is now clear that the cache size may have a major impact on the overall performance of a CCN even if finding an optimal caching strategy is still an open problem to address. With respect to congestion control issues, instead, recent studies shown as the classic additive increase multiplicative decrease algorithm, at the foundation of TCP, could be inherited by $\mathrm{CCN}$, provided that some countermeasures are employed to limit unfairness issues that could arise among contents with different popularities [11], [12]. Another very relevant topic in $\mathrm{CCN}$ research covers routing operations, which are essential to properly drive the dissemination of receiver generated Interest packets. To this end, the adoption of Bloom filters appears a promising solution [13], [14] that merits further investigations. Starting from this premise, it is evident that all facets of $\mathrm{CCN}$ are going to be afforded in an ebullient panorama of activities that cover both the underlying mechanisms within the protocol architecture and the design of content oriented applications and services. With reference to application-related features, it is worth to notice that the demand for TV services is growing at an exponential rate over the time [2], thus requiring a very careful analysis of their performance in $\mathrm{CCN}$ architectures. A preliminary study presented in [15] addresses time shifted applications only, whereas live streaming operations are currently under investigation as testified in the interesting contribution [16]. To complement the research efforts of the community in a so relevant domain, the present manuscript is intended to design a complete CCN-TV system encompassing all the main facets of typical live streaming video services. The proposed CCN-TV has been tested through a solid simulation campaign based on real topologies. To this end, the ccnSim simulator [17] has been properly tailored to our purposes by adding window based flow control, handling of playout delay and real-time data, advanced logging functions, links with bandwidth constraints, and data session bootstrapping. Simulation results shown that in-network caching seems to play a minor role in live streaming video services, mainly because cached data looses its utility after the deadline is expired. On the other hand, the way $\mathrm{CCN}$ handles client requests for TV contents helps improving the performance of the system with respect to a plain IP infrastructure. 
The rest of the paper is organized as follows: Sec. II illustrates the core concepts of CCN. Sec. III describes the CCN-TV system we propose in this paper, which is then evaluated in Sec. IV. Finally, Sec. V summarizes the main findings of this contribution and draws future research.

\section{BASIC BACKGROUND ON CCN}

Internet usage has undergone a radical change during the last ten years: content-sharing applications are now dominant whereas the IP architecture still provides a connection-less service among remote hosts [3]. Users ask for contents, looking for what they intend to retrieve from the Internet while the language spoken by the underlying IP infrastructure provides answers on where a packet should be sent. This mismatch is actually overcome by a number of workarounds at different levels of the protocol stack, which, indeed, limit the overall efficiency of the Internet.

The so-called Future Internet represents a family of possible solutions to the aforementioned issues, embracing novel communication models that can better accommodate and fulfill users' requirements related to efficiency, security, support to mobility, and integrated media experience [3].

At the present stage, many valid proposals for the Future Internet exist, such as the Publish Subscribe Internet Routing Paradigm, the 4WARD NetInf project, and the Cache-andForward Network Architecture, the Data-Oriented Network Architecture and the CCN approach [4], [18], having different levels of compatibility with the IP paradigm.

Among them, the $\mathrm{CCN}$ vision looks promising since, besides being "data-centric", it can be gracefully integrated with today's IP-based Internet. In a $\mathrm{CCN}$, all content is unambiguously identified by a hierarchical name, allowing users to retrieve information without being aware about the physical location of servers (e.g. IP address). Also, communication is receiver-driven and based on content chunk exchange, name-based routing, and self-certifying packets [4].

Nevertheless, the real performance bounds of a $\mathrm{CCN}$ and the actual benefits it can bring to the Internet are still not entirely known, mainly because there are many open issues that surround the $\mathrm{CCN}$ architecture, such as those related to: (i) routing, (ii) congestion control, (iii) strategy layer design, (iv) name space definition, (v) semantic layer, (vi) accurate models, and (v) fairness among heterogeneous applications and contents having different popularities.

As specified before, $\mathrm{CCN}$ communications are driven by the consumer of data and only two types of messages are exchanged (namely Interest and Data). A user may ask for a content by issuing an Interest, which is routed within the $\mathrm{CCN}$ towards the nodes in posses of the required information, thus triggering them to reply with Data packets.

The routing operations are performed by the strategy layer only for Interest packets. Data messages, instead, just follow the reverse path to the requesting user, allowing every intermediate node to cache the forwarded content.
CCN adopts a hierarchical structure for names, which leads to a name tree. In particular, it is formed by several components, each one made by a number of arbitrary octets (optionally encrypted), so that every name prefix identifies a sub-tree in the name space. An Interest can specify the full name of the content or its prefix, thus accessing to the entire collection of elements under that prefix.

Finally, since contents are exchanged based on their names, multiple nodes interested in a particular data can share it using multicast suppression techniques over a broadcast medium. Analyzing a $\mathrm{CCN}$ node, it is possible to identify three main structures [4].

- the Content Store (CS): a cache memory that can implement different replacement policies as Least Recently Used (LRU) and Least Frequently Used (LFU);

- the Forwarding Information Base (FIB): is similar to an IP FIB except for the possibility to have a list of faces $^{1}$ for each Content Name entry, thus allowing Interest packets to be forwarded towards many potential sources of the required Data;

- the Pending Interest Table (PIT): is a table used to keep track of the Interest packets that have been forwarded upstream towards content sources, combining them with the respective arrival faces, thus allowing the properly delivery of backward Data packets sent in response to Interests.

When an Interest packet arrives to a $\mathrm{CCN}$ node, the CS is searched to discover whether a data item is already available as an answer to be sent immediately back to the requesting user. Otherwise, the PIT is consulted to find out if others Interest packets, requiring the same content, have been already forwarded towards potential sources of the required data. In this case, the Interest's arrival face is added to the PIT entry. Otherwise, the FIB is examined to search a matching entry, indicating the list of faces the Interest has to be forwarded through. At the end, if there is not any FIB entry, the Interest is discarded.

On the other hand, when a Data packet is received, the PIT table comes into play, which, keeping track of all previously forwarded Interest packets, allows to establish a backward path to the node that requested the data.

\section{CCN-TV ARCHITECTURE}

Unlike Video-On-Demand, real-time video distribution has to deal with a specific class of problems to ensure the timely delivery of an ordered stream of chunks. Video chunks have to be received in playing order and within a given time interval (the playout delay), before they are actually played, thus "expiring". A chunk not delivered before its expiration will result in degradation of the rendered

\footnotetext{
${ }^{1}$ In $\mathrm{CCN}$ it is used the term "face" instead of the "interface" because packets are also exchanged between application process, besides being forwarded only over real network interfaces.
} 
video, impacting the end user Quality of Experience; the extent of the video degradation may vary depending on the video codec and the type of the lost frame. To solve these challenges, client nodes implement a receiving buffer queue where the chunks are stored in order, that is emptied while the video is being played. Therefore, any chunk not received before its playing instant becomes useless. To reduce the chance of chunk loss several mechanisms can be put in place to retransmit requests for chunks close to expiration. Furthermore, in modern codecs, such as H.264 [19], there are different types of video frames, encoded using intraframe or inter-frame techniques, each having a different level of importance. For example, the so called I-frames, derived using intra-frame compression techniques, actually represent a full video image, providing a fundamental reference for subsequent inter-frame encoded images.

With this in mind in CCN-TV we considered a network of nodes requesting different real-time video streams, identified by a channelID, served by one or more broadcast server.

Unlike canonical UDP/TCP-based streaming, in CCN-TV each video chunk, identified by a progressive chunk number, has to be requested individually, via a dedicated Interest.

Although this might look costly at a first sight, CCN's routing mechanisms ensure that Interests for the same chunk do not propagate twice along the same routing path (unless under specific conditions, as explained in Sec. III-B), and the caching strategy implemented by every node can make sure that Interests for the most popular contents are served before going through the whole routing path. Moreover, the Interest/data exchange allows for a natural flow control mechanism, where each node can request for new chunks just when the old ones have arrived.

Herein we thoroughly describe the design rationale and all the details of the CCN-TV system this paper targets. Specifically, in what follows, we present: the bootstrap phase, the flow control strategy, and the management of retransmitted Interest packets.

\section{A. Channel bootstrap}

One challenge we are faced with in a real-time scenario is to bootstrap the channel to be received. Bootstrapping a channel involves the operations of finding a routing path to the nearest channel provider and locating the first valid chunkID of the video stream. Due to video codec requirements, the video stream can be visualized only once the first I-frame has been received. Therefore, a client has to first gather from the server the first chunk (and the corresponding chunkID) of the last generated I-frame. To do so, it sends an Interest packet for the URI: [domain] / [channelID], with the Status field set to BOOTSTRAP and the Nonce field set by the client, as in in Sec. III-D. An Interest with Status $=$ BOOTSTRAP would travel unblocked until it reaches the first good stream repository (i.e. a node who can provide a continuous real-time flow of chunks, not just cached ones).

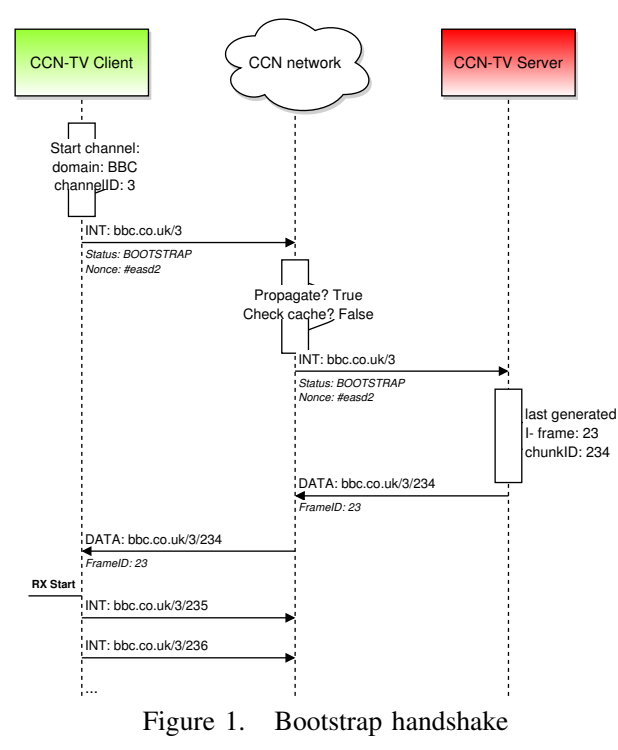

To this Interest, the server responds with a data message in the format [domain]/[channelID]/[chunkID], with chunkID being the first chunk of the last generated I-frame, and the corresponding Frame ID field value. Upon receipt, the node starts asking for subsequent chunks, using the sliding window mechanism detailed in Sec. III-B. The use of a nonce (a uniquely generated identifier) in the Interest URI allows the Interest to propagate to the server without being blocked along the routing path, as every bootstrap Interest for the same channel has a different nonce. It also avoids the retrieval of the data response from the cache of an intermediate node; the risk, in this case, is the retrieval of a bootstrap data message for a given channel from a cache containing an already expired chunk of an I-frame.

\section{B. Flow control}

From the moment a node receives the bootstrap data message, it can initiate the sliding window mechanism to request the subsequent chunks in an optimal way. Each node has a windows of size $W$ to store $\mathrm{W}$ pending chunk. We define pending chunk a chunk whose Interest has been sent by the node, and the window containing the pending chunks a Pending Window. Together with the chunkID, we store in the pending window other information, such as the timestamp of the first request and the timestamp of the last retransmission. Whenever a new data message is received, the algorithm described in Fig. 2 runs over the Pending Window, to perform the following operations:

1) Purge the Pending Window from all the chunks who are expired, i.e., who have already been played, to free new space in the sliding window.

2) Retransmit all chunks that have not been received for a given timeout (onward denoted as windowTimeout.

3) Transmit, for each slot that got freed by the received or expired chunks, the Interest for a new one. 


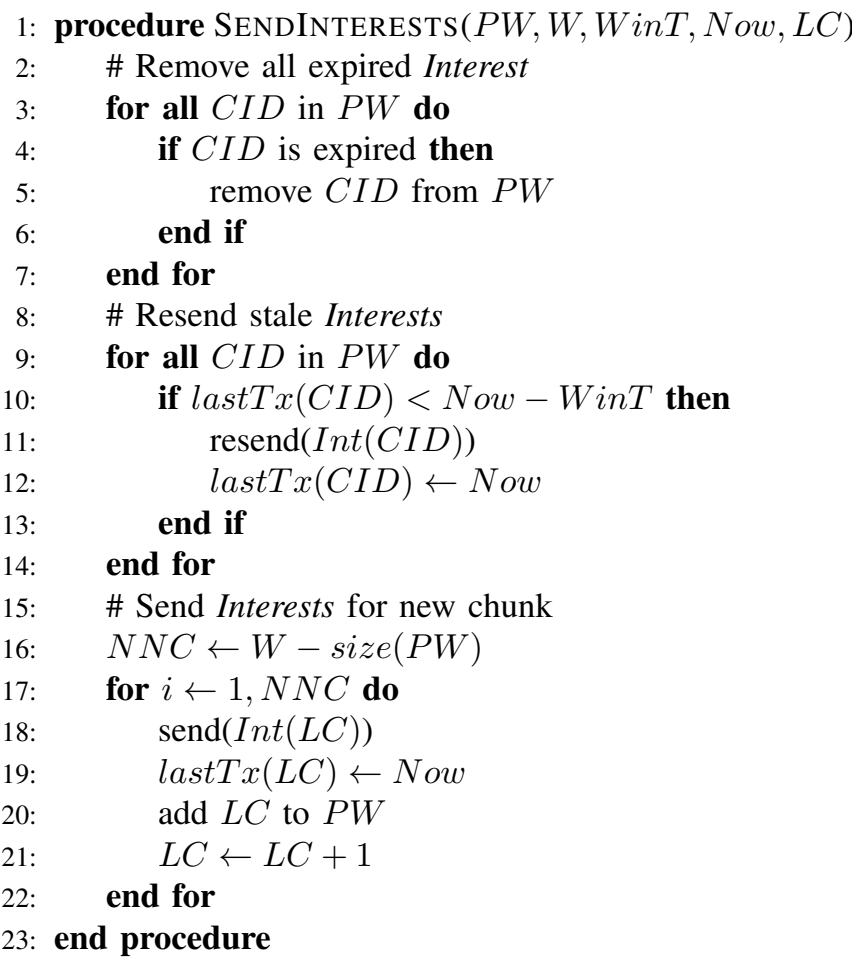

Figure 2. Sliding window algorithm

Furthermore, the same operations are performed if a node doesn't receive any data for at least windowTimeout seconds, in which case, all the Interests for non-expired chunks in the Pending Window are retransmitted, together with new chunks if new slots have been freed due to expired chunks.

Fig. 2 details the implemented algorithm; for the purpose of brevity and readability, the variable names have been contracted: $P W$ is the Pending Window, $W$ is the aforementioned system parameter, indicating how many Interests should a node have ongoing, WinT is the window timeout, after which Interests in the Pending Window are resent, Int is a new Interest message, $C I D$ is a chunkID in the pending window, lastTx is the transmission time of the most recent Interest for a given chunkID, $L C$ is the chunkID of the most recent requested chunk and $N N C$ is the number of new chunks to request, after the pending window has been purged.

To provide a further insight, we reported in Fig. 3 an example of the conceived sliding window algorithm, in which we have set the value of $W$ to be equal to 3 .

\section{Interest routing}

As described in Sec. II, CCN nodes along the routing path of an Interest will stop the propagation of said Interest, if they have previously routed another Interest for the same resource, and the correspondent data has note been sent back yet; instead, they will simply update their Pending Interest Table adding the face from where this newcomer Interest was originated, so to reroute the data back recursively along the path the Interest has gone through.

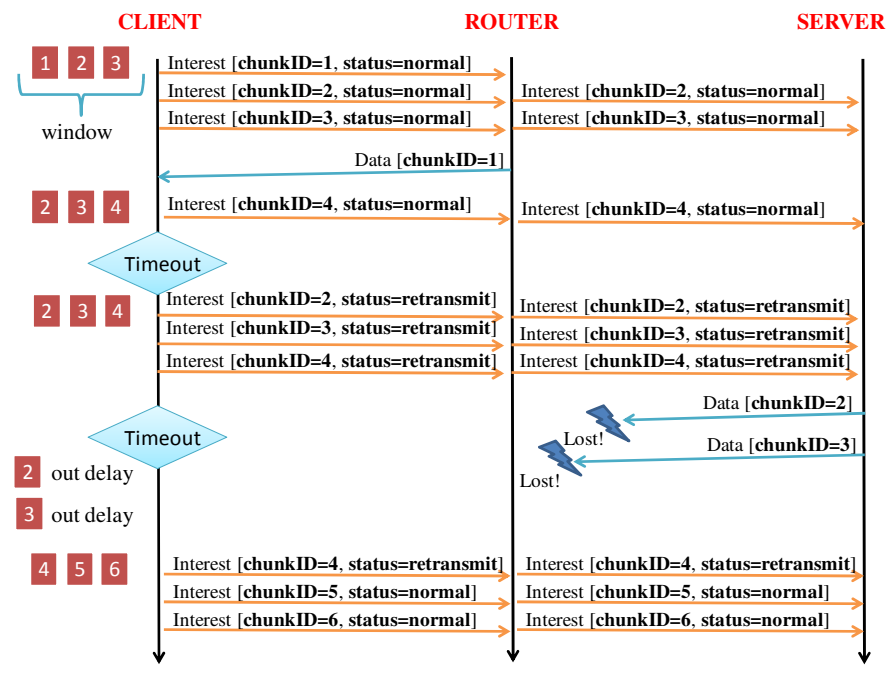

Figure 3. Sliding window example

This mechanism ensures flow control and limits the propagation of duplicate Interests, in case several nodes in the same network are watching the same channel.

However, to make the Interest retransmission mechanism effective, a retransmitted Interest needs to propagate all the way up to the server, or to the first node with the desired chunk in cache. Therefore, retransmitted Interests carry the Status field set to Retransmission to mark if the Interest is a retransmission or not, and each node along the routing path propagates the Interests marked as retransmitted, thus skipping the usual $\mathrm{CCN}$ mechanism, unless the correspondent chunk is found in the cache.

Table I

Messages USED IN CCN-TV

\begin{tabular}{l|l|l}
$\begin{array}{l}\text { Packet } \\
\text { type }\end{array}$ & Field & Content \\
\hline \hline \multirow{4}{*}{$\begin{array}{l}\text { Chunk } \\
\text { Interest }\end{array}$} & $\begin{array}{l}\text { Content } \\
\text { Name }\end{array}$ & {$[$ domain]/[channelID]/[chunkID]. } \\
\cline { 2 - 3 } & nonce & Used only for the bootstrap phase. \\
\cline { 2 - 3 } & $\begin{array}{l}\text { Publisher } \\
\text { Filter }\end{array}$ & Not used. \\
\cline { 2 - 3 } & Status & Bootstrap, Normal, Retransmission. \\
\hline \multirow{4}{*}{$\begin{array}{l}\text { Chunk } \\
\text { Data }\end{array}$} & $\begin{array}{l}\text { Content } \\
\text { Name }\end{array}$ & {$[$ domain]/[channelID]/[chunkID]. } \\
\cline { 2 - 3 } & $\begin{array}{l}\text { Publisher } \\
\text { ID }\end{array}$ & Optional. \\
\cline { 2 - 3 } & $\begin{array}{l}\text { Signature } \\
\end{array}$ & Optional (for increased content authentication). \\
\cline { 2 - 3 } & $\begin{array}{l}\text { Stale } \\
\text { Time }\end{array}$ & $\begin{array}{l}\text { Set to the frame time of the frame the chunk } \\
\text { belongs to. }\end{array}$ \\
\cline { 2 - 3 } & $\begin{array}{l}\text { Frame } \\
\text { ID }\end{array}$ & ID of the frame the chunk belongs to. \\
\cline { 2 - 3 } & Data & The request video chunk binary data. \\
\hline
\end{tabular}

\section{CCN-TV messages}

As detailed above, additional functionalities required by the system for real-time video streaming are implemented on top of existing $\mathrm{CCN}$ data and Interest messages via new fields carrying the required additional information. 
However, should the situation require the system to conform to classical CCN messages, all additional fields can be easily replaced by additional fields in the content name.

Tab. I shows how we made use of the classical CCN message fields, together with the new fields and their use.

In particular, CCN-TV Interests carry an additional Status fields marking if the Interest is a bootstrap Interest (Section III-A), a normal one or a retransmission (Section III-C).

Concerning $\mathrm{CCN}$ data message, we extended the messages with an additional field, i.e., Frame ID, containing the ID of the frame to which the embedded chunk belongs to.

\section{Simulation RESUlts}

In this section, we will evaluate performances of the proposed CCN-TV architecture. To this end, we implemented it within ccnSim, i.e., an open source and scalable chunklevel simulator of CCN [17] built on top of the Omnet++ framework [20], dedicated to the evaluation of Video On Demand systems on top of CCN. By itself, ccnSim models a complete video distribution systems, with a high degree of fidelity concerning catalogs, requests and repositories distribution, and network topologies. Since, however, it did not support the real-time constraints required by our evaluations, it has been modified and improved in the following ways:

- we added support for links with bounded capacity and packets with a well defined size, which was missing in ccnSim, to be able and estimate the $\mathrm{CCN}$ behavior under some bandwidth constraints;

- due to the datarate channels, we implemented a transmission queue for each face of each node, in order to properly manage the packet transmission;

- we added the support for synthetic video traces, so to be able to transmit and receive chunk of real videos, and consequently being able to reconstruct the received video and evaluate its PSNR;

- due to possible expiration of Interests, we implemented a cleanup mechanism for each node's PIT, to avoid having in long term stale entries due to expired chunks;

- we improved and enriched the logging system, so to be able to record each node's received chunks and reconstruct the received video;

- we added more controls server-side, to send a data only for those chunks who have already been generated.

Furthermore, the following mechanisms, beyond the provided ones, have been implemented in the simulator:

- the sliding window mechanism described above, and all the related data structures;

- the Interest forceful propagation in case of retransmission;

- constant data reception, until a channel is changed.

The extended ccnSim simualtor is available at [21].

The aim of our study is to evaluate how the behavior of the CCN-TV system is influenced by (i) the amount of the network bandwidth dedicated to real-time streaming services, (ii) the windowTimeout adopted by the sliding window mechanism, (iii) the playout delay, and (iv) the cache decision policy.

We focus the attention on the GEANT network, which interconnects the European research and education institutions and it is composed by 22 routers [22]. Every node of the network is considered to be a direct $\mathrm{CCN}$ node, i.e. no TCP or UDP encapsulation is implemented. We assume the presence of only one small video streaming provider that offers 5 parallel real-time transmissions to remote clients. It is connected to one of the nodes forming the GEANT topology. In every simulation round, each video content is mapped to a video stream compressed using H.264 [19] at a average coding rate randomly chosen in the range [250, 2000] kbps. Clients, i.e., CCN nodes that download video contents from the server, are connected to remaining nodes ( 1 client per node). In order to catch the behavior of people watching TV, we modeled two groups of users: faithful and zapping. Faithful users are attached to one video channel for the whole simulation. Zapping users, instead, change frequently the channel among those offered by the server according to a Poisson process with parameter $\lambda=0.0666$. Further, the channel selection process has been modeled considering that contents popularities follow a Zipf distribution. According to [23], the most of works presented in literature set the parameter $\alpha$ of the Zipf distribution in the range $[0.6,2.5]$. In line with these common settings, we set $\alpha=1$. Once a client decides to watch a specific channel, it performs the bootstrap process described in the previous section and then starts sending Interest packets following the designed sliding window mechanism.

In our tests, we adopted the optimal routing strategy, already available within the ccnSim framework. According to it, Interest packets are routed towards the video server along the shortest path. On the other hand, three caching strategies have been considered in our study: no-cache, $L R U$, and FIFO [23]. When well known $L R U$ or FIFO policies are adopted, we set the size of the cache to 100 chunks. The no-cache policy is intended to evaluate the performance of the $\mathrm{CCN}$ without using any caching mechanism.

The window size $W$ has been set to 10 , ensuring that faces of the server are almost fully loaded in all considered scenarios. Also, the transmission queue length associated to each face, $Q$, has been set, in order to be larger than

$$
Q=2 * L_{c} * P_{D}
$$

where $L_{c}$ and $P_{D}$ represent link capacity and maximum propagation delay in the considered network topology. All simulation parameters have been summarized in Tab. II.

\section{A. Interest generation process}

As a first step, we investigate the impact that the sliding window mechanism has on the amount of sent Interest 
Table II

SUMMARY OF SIMULATION PARAMETERS

\begin{tabular}{l|l}
\hline Parameter & Value \\
\hline Topology & GEANT with 22 routers \\
Link capacity & $40 \mathrm{Mbps}$ and $100 \mathrm{Mbps}$ \\
Number of real-time service pro- & 1 \\
vides & 21 \\
Number of clients & 5 files \\
Catalog size & $10 \mathrm{Kbytes}$ \\
Chunk size & $250 \mathrm{kbps}, 600 \mathrm{kbps}, 1000 \mathrm{kbps}$ and \\
Video average bit rate & $2000 \mathrm{kbps}$ \\
& 10 \\
W (window size) & $10 \mathrm{~s}$ and $15 \mathrm{~s}$ \\
Playout delay & $1 \mathrm{~s}, 3 \mathrm{~s}$, and $5 \mathrm{~s}$ \\
Window timeout & No cache, LRU, and FIFO \\
Caching strategy & 100 chunks \\
Cache size & $50 \%$ fixed, 50\% changing on aver- \\
Client zapping behavior & age every $15 \mathrm{~s}$ \\
Simulation time & $60 \mathrm{~s}$ \\
Number of seeds & 5 \\
\hline
\end{tabular}

packets, which is shown in Fig. 4. From these plots it is evident that the highest windowTimeout, the lowest the total number of Interest messages sent by end users. When the windowTimeout increases, the probability that a given client does not receive any chunks within such a time interval decreases and, as a consequence, also the number of retransmitted Interest packets decreases as well. As a further confirm of this result, Fig. 5 shows that the percentage of duplicated Interest packets increases when the windowTimeout decreases due to a high number of chunks that are unable to reach the client within the expected timeliness.

As expected, the playout delay has a minor impact on the number of generated Interest messages, which, as is known from the theory on sliding window mechanisms [24], can be only influenced by window size $(W)$ and windowTimeout.

Also, caching operations do not have any significant impact on the number of generated Interest messages. The main reason being that chucks stored in cache memories lose their effectiveness after their deadline is expired.

On the other hand, the link capacity greatly influence the Interest transmission rate. From Fig. 4 emerges, in fact, that the number of Interest lowers when the capacity of links decreases. This is because a limited bandwidth reduces the quota of received chunks, thus preventing a rapid advancement of the sliding window. In other terms, this result proves, once again, the effectiveness of the sliding window mechanism in $\mathrm{CCN}$.

\section{B. QoS and $Q o E$}

The first important parameter that describes how CCNTV settings affect the quality of service offered to end users is the chunk loss ratio, which represents the percentage of chunks that have not been received in time (i.e., before the expiration of the playout delay) by clients.

From Fig. 6, showing the chunk loss ratio measured in all considered network scenarios, we note that playout delay plays a fundamental role. When the playout delay increases, in fact, the client could receive a Data packet within a longer time interval, thus reducing the amount of chunks discarded because out of delay. On the other hand, a slight increment of the chunk loss ratio can be registered by increasing the windowTimeout. If the client retransmits an Interest packet after long time, there is the risk that the Data packet will be reached by the destination after the expiration of the playout delay. In addition, we note that a reduction of the link capacities leads to a higher number of lost chunks, due to increased latencies induced by network congestion.

It is very important to remark that the presence of the cache can guarantee only a small reduction of the chunk loss ratio. With our study, we found that, in the presence of real-time flows, the cache does not represent an important $\mathrm{CCN}$ feature. On the other hand, we noticed that the PIT plays a more relevant role. In fact, in presence of live video streaming services, clients that are connected to a channel request the same chunks simultaneously. In this case, a CCN router has to handle multiple Interest messages that, even though sent by different users, are related to the same content. According to the CCN paradigm, such a node will store all of these requests into the PIT, waiting for the corresponding Data packet. As soon as the packet is received, the router will forward it to all users that have requested the chunk in the past. According to these considerations, the use of the cache will not produce a relevant gain of network performances. Indeed, the PIT helps reducing the burden at the server side by avoiding that many Interest packets for the same chunk are routed to the server.

To conclude our study, we have computed Peak Signal to Noise Ratio (PSNR), which is nowadays one of the most diffused metrics for evaluating user satisfaction, together with interactivity level, in real time video applications [25]. Results shown in Fig. 7 are in line with those reported for chunk loss ratio (the PNSR is higher in the same case in which the chunk loss ratio is lower). Again, link capacity greatly influences the quality of the TV service provided to users. According to [26], the obtained PSNR values can be translated to a Mean Opinion Score (MOS) not less than 4, corresponding to satisfactory quality for almost all users.

\section{CONCLUSIONS AND FUTURE RESEARCH}

In this work, the effectiveness of TV services in a CCN has been investigated. To this end, the ccnSim simulator has been modified to add several relevant features such as window-based flow control, handling of playout delay and real-time data, advanced logging mechanisms, and data session bootstrapping. Preliminary results reported herein clearly show that the most relevant $\mathrm{CCN}$ feature to TV services is the management of Interest packets through the PIT data structure. In fact, such a mechanism limits the number of requests for the same chunk at the server side for multiple clients watching the same TV channel, thus decreasing 

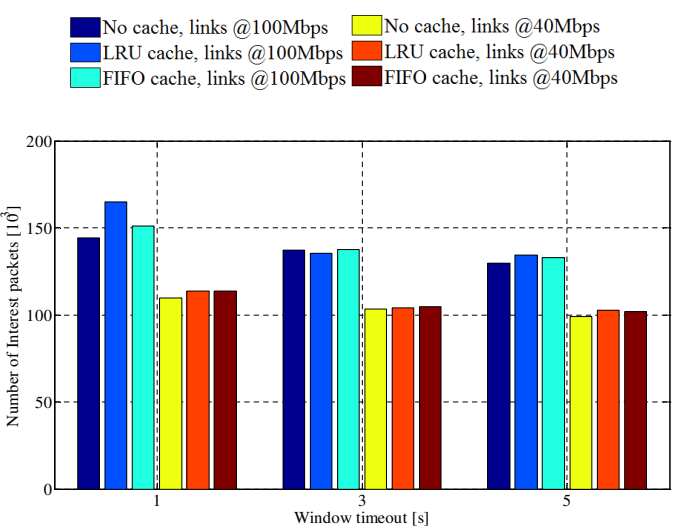

(a)

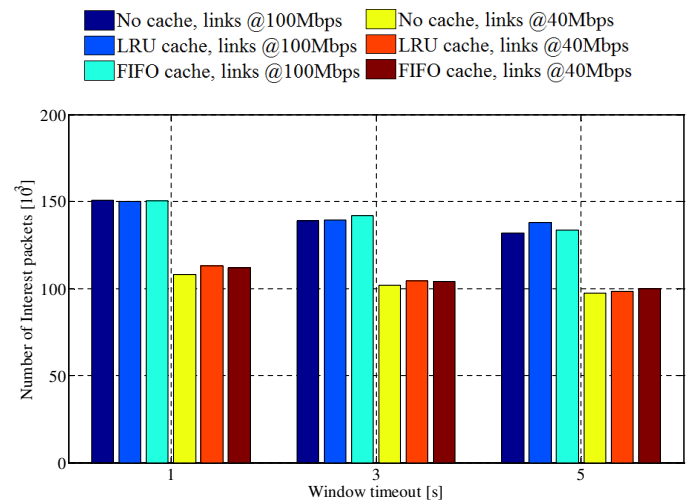

(b)

Figure 4. Total number of Interest packets sent by clients with playout delay of (a) $10 \mathrm{~s}$ and (b) $15 \mathrm{~s}$.

the link and the computational load at the server. Further research will explore: wider scenarios with many users and available channels, advanced optimization techniques for TV services in CCN (including routing and congestion control), and the adoption of scalable video coding. Moreover, a more deeply investigation on the relevance of the cache and the PIT in live TV services will be also conducted.

\section{REFERENCES}

[1] H. Kopka and P. W. Daly, A Guide to $B T_{E} X$, 3rd ed. Harlow, England: Addison-Wesley, 1999.

[2] "Cisco visual networking index: Forecast and methodology, 2010-2015,” White Paper, Cisco, Jun. 2011.

[3] B. Ahlgren, P. A. Aranda, P. Chemouil, S. Oueslati, L. M. Correia, H. Karl, M. Sollner, and A. Welin, "Content, connectivity, and cloud: ingredients for the network of the future," IEEE Commun. Mag., vol. 49, no. 7, Jul. 2011.

[4] V. Jacobson, D. K. Smetters, J. D. Thornton, M. F. Plass, N. H. Briggs, and R. L. Braynard, "Networking named content," in ACM CoNEXT '09, 2009.

[5] G. Carofiglio, M. Gallo, L. Muscariello, and D. Perino, "Modeling data transfer in content-centric networking," in Int. Teletraffic Congress, (ITC), 2011.

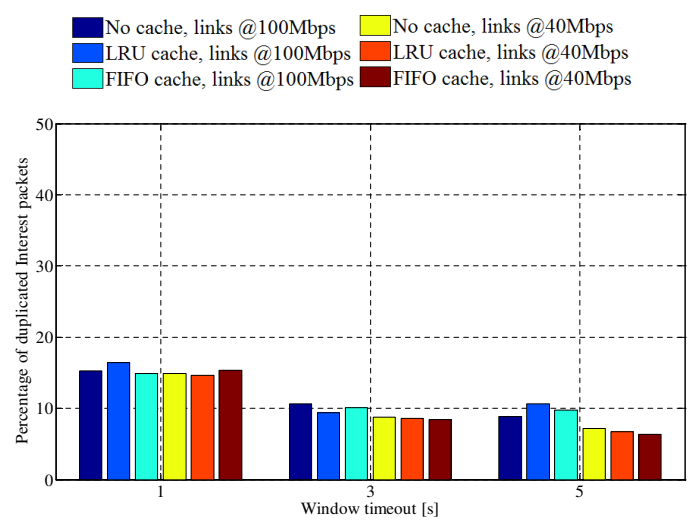

(a)

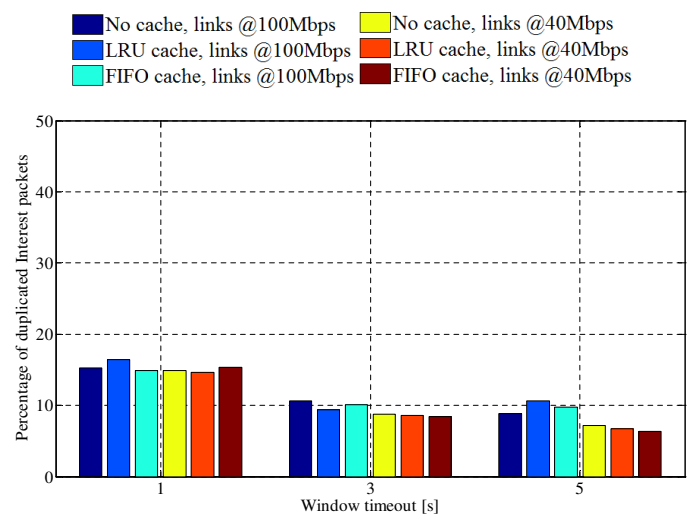

(b)

Figure 5. Percentage of duplicated Interest packets sent by clients with playout delay of (a) $10 \mathrm{~s}$ and (b) $15 \mathrm{~s}$.

[6] L. Muscariello, G. Carofiglio, and M. Gallo, "Bandwidth and storage sharing performance in information centric networking," in ACM SIGCOMM workshop on Information-centric networking (ICN'11), 2011.

[7] M. Varvello, I. Rimac, U. Lee, L. Greenwald, and V. Hilt, "On the design of content-centric manets," in Int. Conf. on Wireless On-Demand Network Systems and Services, (WONS), 2011.

[8] G. Carofiglio, V. Gehlen, and D. Perino, "Experimental evaluation of memory management in content-centric networking," in IEEE ICC, 2011.

[9] M. Tortelli, I. Cianci, L. A. Grieco, G. Boggia, and P. Camarda, "A fairness analysis of content centric networks," in Proc. of Int. Conf. on Network of the Future, NOF, Paris, France, 2011.

[10] D. Rossi and G. Rossini, "On sizing CCN content stores by exploiting topological information," in IEEE INFOCOM, NOMEN Worshop, 2012.

[11] L. A. Grieco, D. Saucez, and C. Barakat, "AIMD and CCN: past and novel acronyms working together in the Future Internet," in Capacity Sharing Workshop 2012 (CSWS'12) colocated with ACM SIGCOMM CoNEXT 2012., Dec. 2012. 


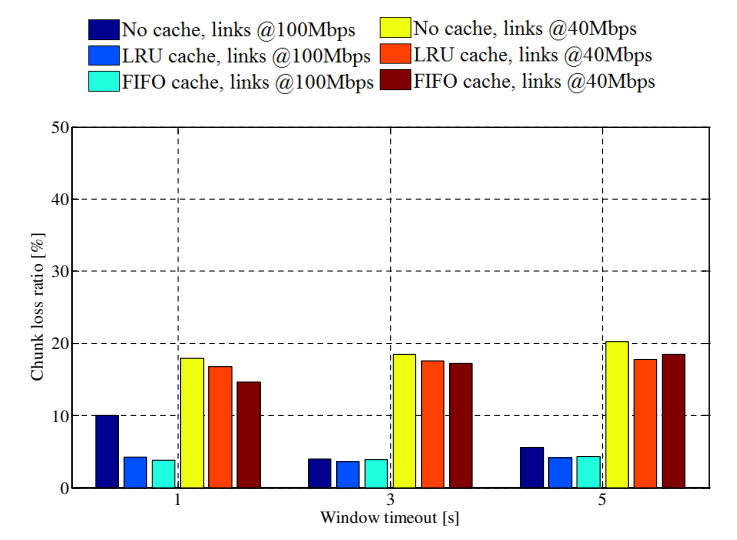

(a)

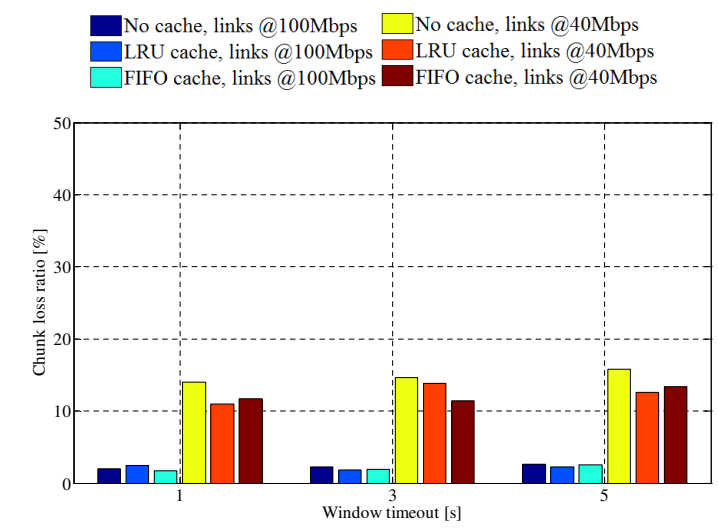

(b)

Figure 6. Chunk loss ratio with playout delay of (a) $10 \mathrm{~s}$ and (b) $15 \mathrm{~s}$.

[12] G. Carofiglio, M. Gallo, and L. Muscariello, "Joint hop-byhop and receiver-driven interest control protocol for contentcentric networks", ACM SIGCOMM, ICN12 workshop, 2012.

[13] M. Tortelli, L. A. Grieco, and G. Boggia, "CCN forwarding engine based on bloom filters," in Proc. of ACM Int. Conf. on Future Internet Technologies, CFI, Seoul, Korea, Sep. 2012.

[14] W. You, B. Mathieu, P. Truong, J. Peltier, and G. Simon, "Dipit: A distributed bloom-filter based pit table for $\mathrm{CCN}$ nodes," in 21st International Conference on Computer Communications and Networks (ICCCN), 2012, pp. 1-7.

[15] Z. Li and G. Simon, "Time-shifted TV in content centric networks:the case for cooperative in-network caching," in Proc. of IEEE ICC, Jun. 2011.

[16] H. Xu, Z. Chen, R. Chen, and J. Cao, "Live streaming with content centric networking," in Proc. 3rd Int. Conf. on Networking and Distributed Computing, Hangzhou, China, 2012.

[17] G. Rossini and D. Rossi, "Large scale simulation of ccn networks," in In Algotel 2012.

[18] B. Ahlgren, C. Dannewitz, C. Imbrenda, D. Kutscher, and B. Ohlman, "A survey of information-centric networking," Communications Magazine, IEEE, 50(7), pp. 26-36, 2012.

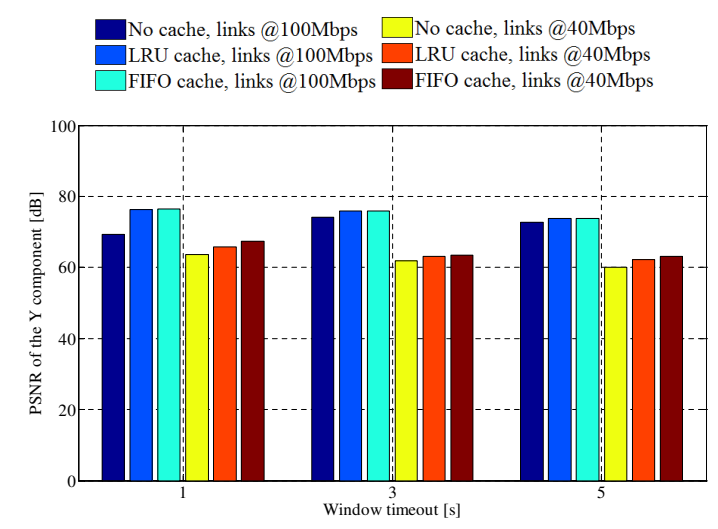

(a)

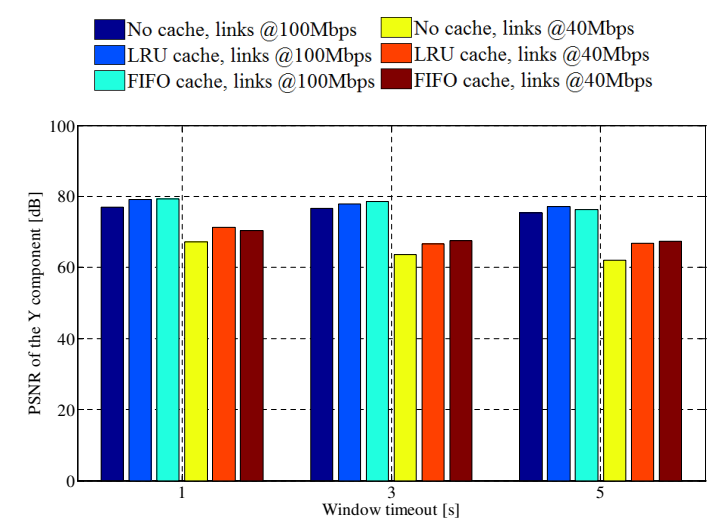

(b)

Figure 7. PSNR of the $\mathrm{Y}$ components of received videos with playout delay of (a) $10 \mathrm{~s}$ and (b) $15 \mathrm{~s}$.

[19] T. Wiegand, G. Sullivan, G. Bjontegaard, and A. Luthra, "Overview of the H.264/AVC video coding standard," IEEE Trans. on Circuits and Systems for Video Technology, 13(7), pp. 560-576, Jul. 2003.

[20] Omnet++ home page. [Online]. Available at: http://www. omnetpp.org/

[21] Ccn-tv webpage. [Online]. Available at: http://telematics. poliba.it/ccn-tv/

[22] "Geant project website," [OnLine] Available at: http://www. geant.net/.

[23] D. Rossi and G. Rossini, "Caching performance of content centric networks under multi-path routing (and more)," in Technical report, Telecom ParisTech, 2011.

[24] J. F. Kurose and K. W. Ross, Computer Networking: A Top-Down Approach, 6th edition. Addison-Wesley Publishing Company, 2012.

[25] G. Piro, L. Grieco, G. Boggia, R. Fortuna, and P. Camarda, "Two-level Downlink Scheduling for Real-Time Multimedia Services in LTE Networks," in IEEE Trans. Multimedia, vol. 13, no. 5, Oct. 2011, pp. 1052-1065.

[26] J. Ohm, Multimedia Communication Technology. Springer, USA, 2004. 